\title{
Clinical and functional significance of CHK1-S, an alternatively spliced isoform of the CHKI gene, in hepatocellular carcinoma
}

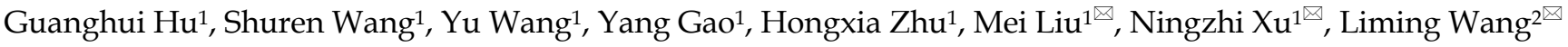 \\ 1. Laboratory of Cell and Molecular Biology \& State Key Laboratory of Molecular Oncology, National Cancer Center/National Clinical Research Center for \\ Cancer/Cancer Hospital, Chinese Academy of Medical Sciences and Peking Union Medical College, Beijing, China. \\ 2. Department of Hepatobiliary Surgery, National Cancer Center/National Clinical Research Center for Cancer/Cancer Hospital, Chinese Academy of \\ Medical Sciences and Peking Union Medical College. \\ $\square$ Corresponding authors: Liming Wang. Department of Hepatobiliary Surgery, National Cancer Center/National Clinical Research Center for Cancer/Cancer \\ Hospital, Chinese Academy of Medical Sciences and Peking Union Medical College, 17 Panjiayuan Nanli, Chaoyang District, Beijing 100021, China. Mobile \\ phone: +86-13161361180; Email: stewen_wang@sina.com; Mei Liu. Laboratory of Cell and Molecular Biology \& State Key Laboratory of Molecular Oncology, \\ National Cancer Center/National Clinical Research Center for Cancer/Cancer Hospital, Chinese Academy of Medical Sciences and Peking Union Medical \\ College, 17 Panjiayuan Nanli, Chaoyang District, P.O. Box 2258, 100021, Beijing, P. R. China. Tel: +8610 87788487; Fax: +8610 67738220; E-mail: \\ liumei@cicams.ac.cn; Ningzhi Xu. Laboratory of Cell and Molecular Biology \& State Key Laboratory of Molecular Oncology, National Cancer Center/National \\ Clinical Research Center for Cancer/Cancer Hospital, Chinese Academy of Medical Sciences and Peking Union Medical College, 17 Panjiayuan Nanli, Chaoyang \\ District, P.O. Box 2258, 100021, Beijing, P. R. China. Tel: +8610 87787369; Fax: +8610 67738220; E-mail: xuningzhi@cicams.ac.cn.
}

(c) The author(s). This is an open access article distributed under the terms of the Creative Commons Attribution License (https://creativecommons.org/licenses/by/4.0/). See http://ivyspring.com/terms for full terms and conditions.

Received: 2019.08.18; Accepted: 2019.12.01; Published: 2020.01.17

\begin{abstract}
Alternative splicing plays critical roles in many disease processes and splicing dysregulation is a hallmark of cancer. The different splicing isoforms may have significantly different effects on the malignant progression of cancer. Checkpoint kinase $1(\mathrm{CHK} 1)$ is a serine/threonine kinase and regulates DNA damage response. In this study, we measured the expression of an alternative $\mathrm{CHK} 1$ transcript (CHK1-S, excluded exon 3) in hepatocellular carcinoma (HCC) tissues. Our results showed that CHKI-S was significantly upregulated in HCC tissues compared with paired adjacent noncancerous hepatic tissues. The levels of full-length CHK1(CHK1-L), CHK1-S and the ratio of CHK1-S/L in tumor tissue were associated with relapse free survival (RFS) of postoperative HCC patients, respectively, but not the levels of CHK1-L, CHK1-S and the ratio of CHK1-S/L in adjacent normal tissue. To further demonstrate the role of CHKl-S in HCC, CCK-8 assays, EdU incorporation assays and colony formation assays were used. The results showed that overexpression of CHKI-S significantly accelerated HCC cell proliferation, compared with CHK1-L. In addition, we found that serine-arginine protein kinase 1 (SRPK1), as an upstream regulator kinase of splicing factor, could upregulate the expression of CHK1-S and its expression level was significantly higher in HCC tumors than the paired normal tissues and was associated with the levels of CHK1-S $(P=0.016)$. In conclusion, our study demonstrated that $\mathrm{CHK} 1-\mathrm{S}$, acts as an oncogene, which was upregulated and associated with RFS in HCC patients. SRPK1 may mediate its mRNA splicing in HCC. All these data indicated that the expression of CHK1-S would have potential prognostic values and splicing kinase SRPK1 might be developed as therapeutic target in HCC.
\end{abstract}

Key words: CHK1, CHK1-S, HCC, SRPK1, RFS

\section{Introduction}

Hepatocellular carcinoma (HCC) is one of the most prevalent primary liver cancer and the major cause of cancer death in China [1, 2]. Though many curative therapies have been developed, the overall response to these therapies leaves much to be desired and the long-term prognosis of HCC patients remains 
poor owing to its high recurrence rates [3]. Deciphering the molecular mechanisms of HCC initiation and progression may give rise to improved diagnostic and therapeutic strategies. Therefore, it is urgently needed to identify reliable early diagnostic biomarkers and effective treatment options.

Alternative splicing generates different mature mRNAs through the inclusion or exclusion of specific exons to increase transcriptome and proteome diversity [4]. Alternative splicing has critical roles in normal development, while its dysregulation is commonly observed in numerous diseases, including cancer [5-7]. Cancer-specific mRNA transcripts, regulated by trans-acting splicing factors, may result in either loss-of-function of tumor suppressor genes or activation of oncogenes, and promote cell proliferation or survival, eventually leading to tumor invasion and metastasis [8]. Induced by amphiregulin, p73 is alternatively spliced into its oncogenic isoform, $\Delta$ Ex2p73, during liver tumorigenesis [9]. Two Numb splice isoforms, which differ in inclusion (long, PRRL) or exclusion (short, PRRS) of exon 12, have opposite effects on HCC cell proliferation [10]. The ${ }^{\triangle 2-4}$ Merlin splice variant that lacks exons 2, 3 and 4 disrupts the normal function of Merlin and promotes hepatocellular carcinoma metastasis [11].

Checkpoint kinase 1 (CHK1) is a serine/threonine protein kinase originally identified as a regulator that controls the $\mathrm{G} 2 / \mathrm{M}$ phase transition in response to DNA damage in yeast [12]. CHK1 plays a critical role in sensing the initial single strand DNA breaks to activate DNA damage response [13]. Activated CHK1 phosphorylates its downstream targets to bring about cell cycle arrest, activate DNA repair pathways, and induce apoptosis when DNA damage is severe [14-17]. Recently, it has been reported that $\mathrm{CHK} 1$ is overexpressed in HCC tissues compared with adjacent non-tumour tissue [18]. In addition, previous study has reported that CHK1-S, an alternative splice variant of CHK1, interacts with CHK1 and acts as an endogenous inhibitor of CHK1 [19]. The study has also showed that CHK1-S expression correlates with the degree of malignancy in ovarian and testicular tumors [19]. However, there has hitherto been a lack of information regarding the status of CHK1-S in HCC.

In this study, we demonstrated that CHK1-S was upregulated in HCC tissues. High CHK1-S (which lacks exon 3) and CHK1-L (full-length CHK1) expression were significantly associated with worse relapse free survival (RFS) of postoperative HCC patients, besides, high ratio of CHK1-S/L in HCC tissues correlated with reduced RFS. CHK1-S functioned as an oncogene in promoting tumor growth. SRPK1 may be the splicing modulator that regulates the alternative splicing of $\mathrm{CHK} 1$ in $\mathrm{HCC}$.

\section{Materials and methods}

\subsection{Tissue samples}

HCC tissues and the paired adjacent noncancerous hepatic tissues were obtained from 54 patients who underwent radical resections in the Cancer Hospital (Chinese Academy of Medical Sciences \& Peking Union Medical College) between May 2012 and April 2015. In this retrospective study, the process does not interfere any diagnosis and treatment, the results will not contain any identifiable patient's information and be published as statistically analyzed data. Moreover, some of the included patients have died and it is impossible to obtain informed consent. Thus, the Ethics Committee approved the study and permitted to waive the informed consent of all the patients in this study (3332018193). The clinical characteristics of the patients were shown in Table 1.

Table 1. Clinical characteristics of $54 \mathrm{HCC}$ patients according to CHK1-S ratio.

\begin{tabular}{llll}
\hline & Low Ratio $(\mathrm{n}=27)$ & High Ratio $(\mathrm{n}=27)$ & P value \\
\hline Gender (Male/Female) & $22 / 5$ & $23 / 4$ & 1.000 \\
Age (years) & $55.04 \pm 10.00$ & $54.07 \pm 13.47$ & 0.767 \\
$\begin{array}{l}\text { Tumor size (cm) } \\
\begin{array}{l}\text { Microvascular invasion } \\
\text { (Present/Absent) }\end{array}\end{array}$ & $4.86 \pm 2.00$ & $5.36 \pm 2.87$ & 0.461 \\
$\begin{array}{l}\text { Differentiation } \\
\text { (Moderate/Poorly) }\end{array}$ & $16 / 11$ & $14 / 13$ & 0.584 \\
$\begin{array}{l}\text { Satellite Nodules } \\
\text { (Present/Absent) }\end{array}$ & $21 / 6$ & $17 / 10$ & 0.233 \\
$\begin{array}{l}\text { Envelope invasion } \\
\text { (Present/Absent) }\end{array}$ & $22 / 5$ & $23 / 4$ & 1.000 \\
$\begin{array}{l}\text { Cirrhosis (Present/Absent) } \\
\text { Ln(AFP) }\end{array}$ & $24 / 4$ & $18 / 9$ & 0.111 \\
& $5.27 \pm 2.82$ & $22 / 5$ & 0.704 \\
\hline
\end{tabular}

\subsection{Plasmids}

The cDNA obtained from 293T cells was used as template to amplify genes of interest in the course of subsequent clonings. Human CHK1 gene (GenBank: NM_001114122.2 or NM_001330428.1) encoding full length or excluded exon 3 were amplified by PCR and subcloned into pcDNA3.1-Myc/His B(-) vector, respectively. All the primers used for cloning were enlisted in Table 2. Human SRPK1 plasmids (pReceiver-M35-C-Flag) were bought from Fulen Gene (Guangzhou, China).

\subsection{Cell cultures}

The human HCC cell lines HepG2 and QSG-7701 were obtained from Cell Resource Center (Beijing, China). The cells were grown in Dulbecco's modified Eagle's medium (DMEM) (Shengwuxigong Company, Beijing ,China) containing $10 \%$ fetal bovine serum (Invitrogen) and maintained at $37^{\circ} \mathrm{C}$ in an 
atmosphere containing $5 \% \mathrm{CO}_{2}$. For construction of stably overexpressed cells, pLVX CHK1-S, pLVX CHK1-L were transfected into HepG2 cells and selected with puromycin $(2 \mathrm{mg} / \mathrm{ml})$ for one week.

\subsection{Real-time PCR}

Total RNAs were extracted using the Trizol reagent (Invitrogen) following the manufacturer's instruction. cDNA was synthesized using PrimeScript ${ }^{\mathrm{TM}}$ RT reagent Kit (Takara, RR047A). PCR reactions were carried out on a StepOnePlus ${ }^{\mathrm{TM}}$ Real-time PCR System (Applied Biosystems, Carlsbad, CA, USA) in $20 \mu \mathrm{l}$ reaction volume. The primers used were enlisted in Table 2. TaqMan-based real-time PCR was conducted for detecting CHK1-L and CHK1-S expression. SYBR-based real-time PCR was conducted for detecting SRPK1 expression.

Table 2. Primer Sequence Information.

\begin{tabular}{ll}
\hline & Primer Sequence 5' ${ }^{\prime}{ }^{\prime}$ \\
\hline pLVX CHK1-S & F-CCGGAATTCATGGAGAAGCCAGACATAGGCA \\
& R-GGGGGGCCTCATGTGGCAGGAAGCCAAATCT \\
pLVX CHK1-L & F-CCGGAATTCATGGCAGTGCCCTTGTGG \\
& R-GGGGGCCCTCATGTGGCAGGAAGCCAAATCT \\
pcDNA 3.1 & F-CCGGAATTCATGGAGAAGCCAGACATAGGCA \\
CHK1-S & R-CGGGGTACCGATGTGCAGGAAGCCAAATCT \\
pcDNA 3.1 & F-CCGGAATTCATGGCAGTGCCCTTGTGG \\
CHK1-L & R-CGGGGTACCGATGTGGCAGGAAGCCAAATCT \\
CHK1-L & F-GGTGCCTATGGAGAAGTCAA \\
& Probe- CAATCTTCACTGCGACTGCTTCTTCAG \\
& R-TCTACGGCACGCTTCATATC \\
CHK1-S & F-GTGCAAACCCTGGGAGAA \\
& Probe-TGCCTATGTCTGGCTTCTCCATAGGC \\
& R-CTCTGAGCATCTGGTTCAGG \\
ACTIN & F-ACCTTCTACAATGAGCTGCG \\
& Probe- ATCTGGGTCATCTTCTCGCGGTG \\
SRPK1 & R-CCTGGATAGCAACGTACATGG \\
& F- CGGTTGCTGAAGTCAGTTCG \\
ACTIN & R-ACTTGAGCAGATGATGCCCC \\
& F-CTACAATGAGCTGCGTGTGGC \\
& R-CAGGTCCAGACGCAGGATGGC \\
\hline
\end{tabular}

\subsection{Antibodies and Immunoblot Analysis}

Extraction of proteins from cultured cells or tissues using RIPA buffer was followed by immunoblot analyses. The following antibodies were used for initial immunoblot analysis. CHK1 (1:1000, 2360, Cell Signaling Technology), SRPK1 (1:1000, 14073-1-AP, Proteintech), beta-Actin (1:1000, 60008-1-Ig, Proteintech). After extensively washed with TBS-T buffer, the membranes were then incubated with secondary antibodies (ZSGB-BIO, China) for $1 \mathrm{~h}$ at room temperature. Protein expression levels were detected using enhanced chemiluminescence (Engreen, China) according to the manufacturer's instructions.

\subsection{Cell proliferation assays}

Cell proliferation was assessed using Cell Counting Kit-8 and Ethynyl deoxyuridine (EdU) incorporation assays. For cell viability, a total of 4000
HepG2 cells were plated in 96-well plates and measured using the CCK-8 (Dojindo Laboratories, Japan) following the manufacturer's instructions. The cell proliferation curves were plotted using the luminescence at each time point. EdU incorporation assays were performed with an EdU kit (Beyotime Company, China) following the manufacturer's instructions. The images were obtained by Confocal Microscope (Leica, Germany).

\subsection{Colony formation assays}

One thousand HepG2 cells were seeded per well in 6-well plates and incubated for 10 days with normal DMEM medium. The colonies were fixed by $0.4 \%$ PFA and stained with $0.5 \%$ crystal violet.

\subsection{Microarray data analysis}

The Affymetrix GeneChip Human Transcriptome Array (HTA2.0) were used to identify differentially expressed genes of paired tumor and non-tumor tissues from five HCC patients with recurrence within 1 year after primary resection. The RNAs were isolated using Trizol reagent. Total RNA was converted to CDNA and then hybridized to Affymetrix HTA 2.0 arrays according to the manufacturer's instructions. The raw data of microarray was pretreated by Expression Console software. We performed a Student's paired t-test to compare gene intensities in the different tissue samples. Genes that had fold changes of $\geq 2.0$ and uncorrected p-value $<0.05$ were considered significantly regulated.

\subsection{Statistical analysis}

For comparisons, Wilcoxon signed-rank test, Student's t-test, and Mann-Whitney test were performed as indicated. $P$-values were obtained with the SPSS 18.0 software (SPSS, USA). $P$-values $<0.05$ were defined as statistically significant.

\section{Results}

\subsection{Clinical significance of splicing isoform CHK1-S in hepatocellular carcinoma}

We examined the expression level of CHK1-L (full-length CHK1) and CHK1-S (the shorter isoform of CHK1, which lacks exon 3) in HCC tumors (T) and paired surrounding nontumor tissues (NT) from 54 patients by real-time PCR, respectively (Fig. 1A). We found that CHK1-S mRNA expression was significantly higher in HCC tumors compared with matched non-tumor tissues (Fig. 1B), and CHK1-S/L ratio (CHK1-S/CHK1-L in mRNA level) was higher in HCC than paired non-tumor tissues (Fig. 1C). Above all, CHK1-S, a splicing isoform of CHK1, was upregulated in HCC tissues. 


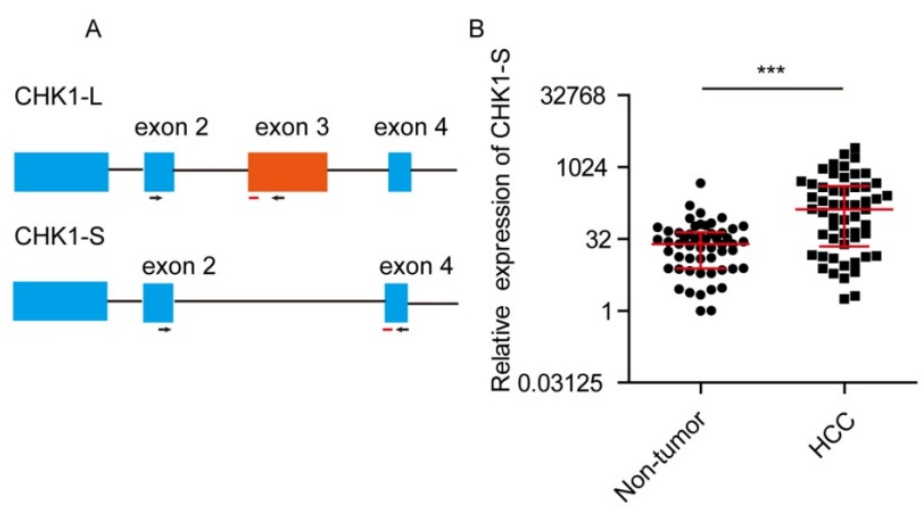

C

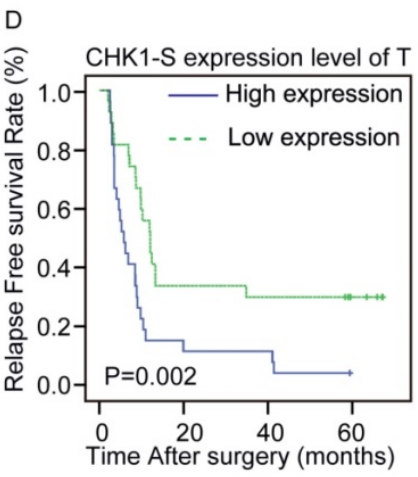

E

$\mathrm{F}$

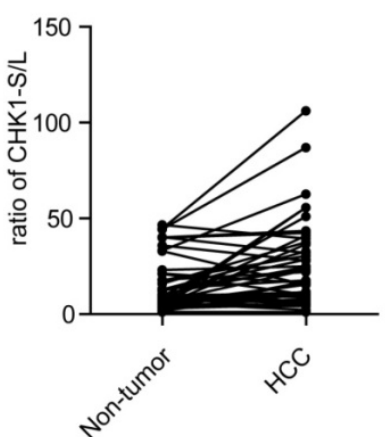

CHK1-L expression level of T

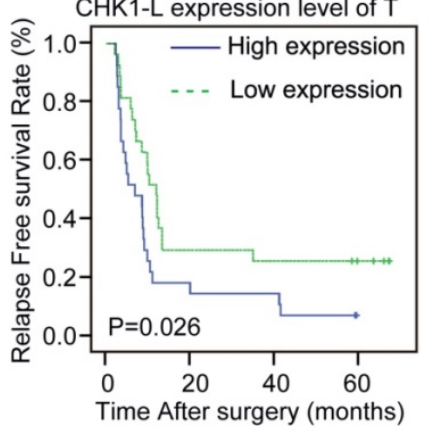

CHK1-S expression level of NT

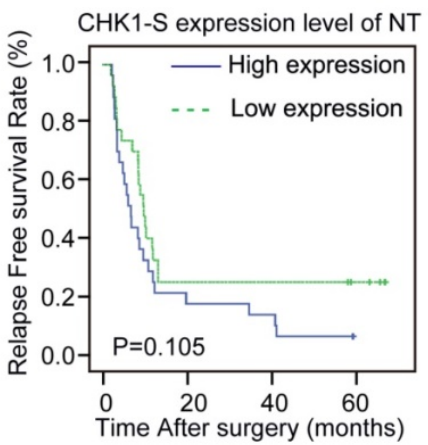

G

$\mathrm{H}$
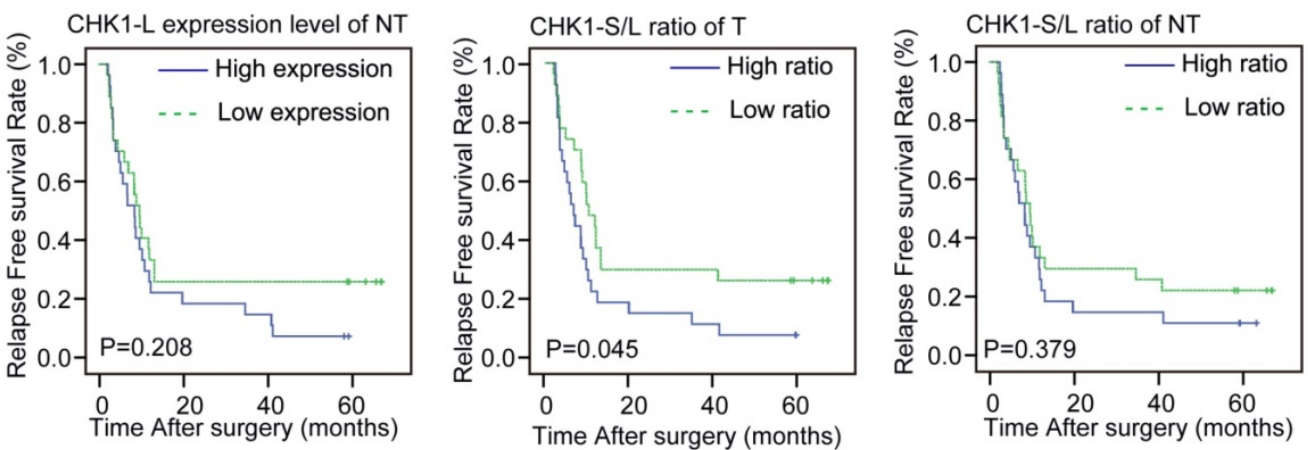

Figure 1. Clinical significance of CHK1-S in HCC. (A) Schematic showing alternative splicing of CHK1. Arrowheads and red lines respectively represent the primers and the probes used for splicing assays with quantitative PCR. (B) CHK1-S expression in human HCC tissues and paired adjacent noncancerous hepatic tissues was examined by quantitative PCR. $n=54, P=0.007$ by Mann-Whitney test. (C)The ratio of CHK1-S/L (CHK1-S/CHK1-L) in 54 paired human HCC tissues and adjacent noncancerous hepatic tissues. The mRNA expression of CHKI-S and CHK1-L were examined by real-time qPCR. A paired two-tailed Student's t-test was used. $P=0.0001$. (D-I) Kaplan-Meier curves depicting relapse free survival (RFS). Expression values or ratios were determined for all HCC samples indicated within the cohort. Samples were stratified based on median expression values or ratios. Plots compare the RFS for CHK1-S mRNA level in tumor tissues (D), CHK1-L mRNA level in tumor tissues (E), CHK1-S mRNA level in adjacent non-tumor tissues(NT) (F), CHK1-L mRNA level in adjacent NT tissues (G) CHK1-S/L ratio in tumor tissues $(\mathrm{H}), \mathrm{CHK} 1-\mathrm{S} / \mathrm{L}$ ratio in adjacent NT tissues (I), respectively. The $P$ values were calculated using the log-rank test.

To investigate the correlation between CHK1-S and clinical characteristics, we divided the 54 patients into two groups based on the median value of the expression ratio of CHK1 S/L. As shown in Table 1, the clinic-pathological features of HCC patients, including the patient's age, gender, tumor size, microvascular invasion, differentiation, envelope invasion, satellite nodules, cirrhosis and AFP, have no significant difference between the low and high CHK1-S/L ratio group $(P>0.05)$.

In order to further study the relationship between $\mathrm{CHK} 1$ isoforms and the prognosis of HCC patients, we made univariate analysis and multivariate analysis. As showed in Table 3, univariate analysis indicated that satellite nodules, microvascular invasion, CHK1-S in tumor tissue, the ratio of $\mathrm{CHK} 1-\mathrm{S} / \mathrm{L}$ in tumor tissues and adjacent nontumor tissues were significantly associated with RFS of HCC patients, whereas other features, including age at diagnosis, gender, differentiation, cirrhosis, and envelope invasion, AFP, CHK1-L in tumor or adjacent normal tissue were not. For multivariate analysis, the ratio of CHK1-S/L in tumor tissues and microvascular invasion were significantly associated with RFS of HCC patients. 


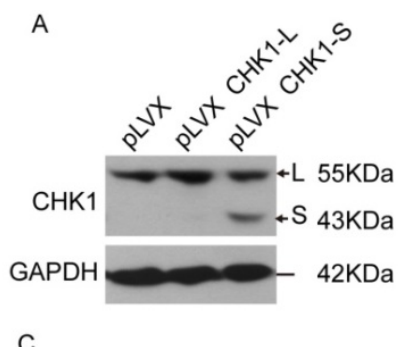

C
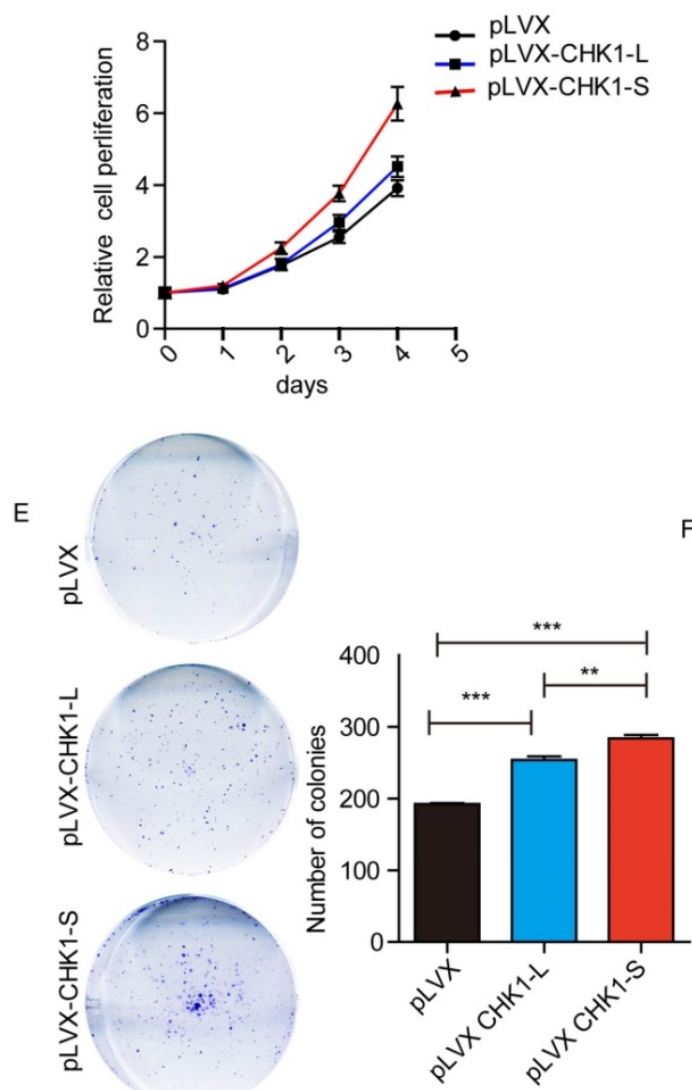

B
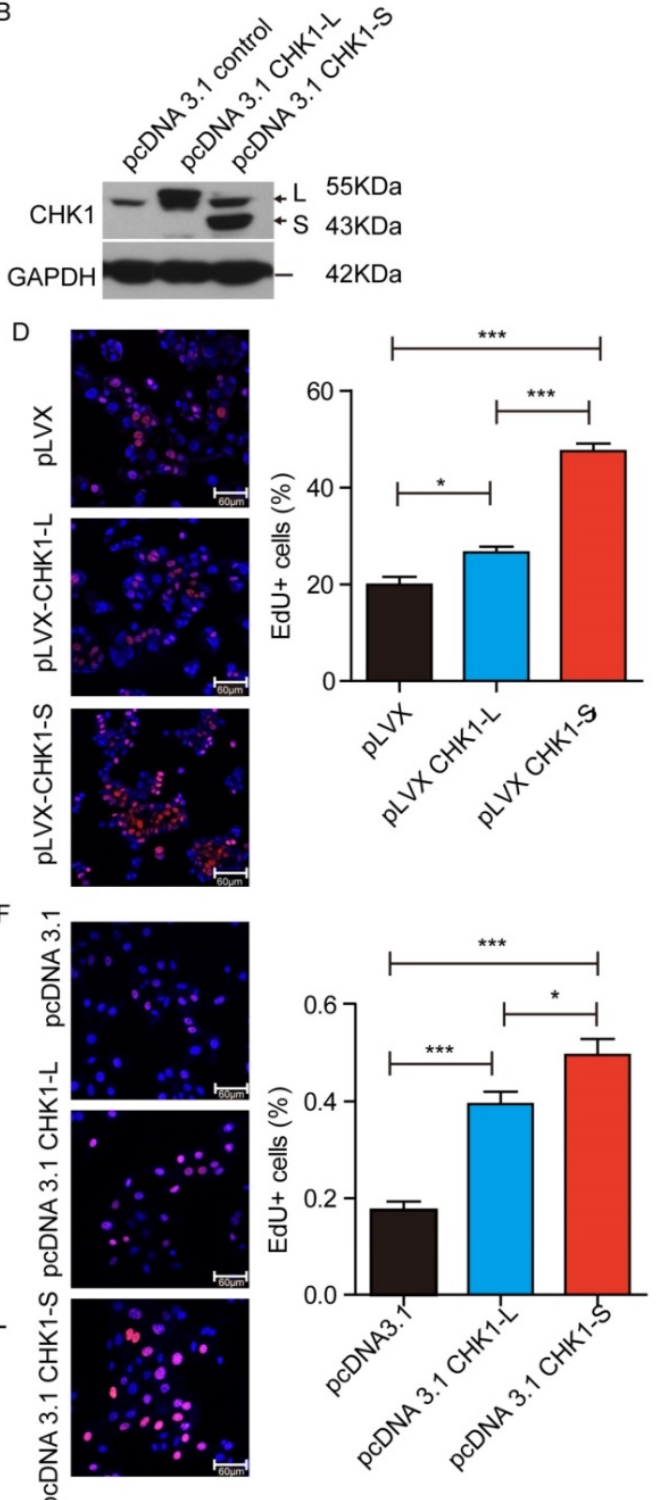

Figure 2. CHK1-S significantly promoted HCC cell proliferation. (A\&B) Overexpression of CHK1-L and CHK1-S in HepG2 or QSG-7701 cells were examined by western blot. HepG2 cells stably transfected with Lenti-viral empty plasmid pLVX and QSG-7701 cells transient transfected with pcDNA3.1 empty plasmid were used as control, respectively. (C) Cell proliferations were measured using CCK-8 assays in HepG2 cells. (D) Colony formation assays of HepG2 cells stably overexpressing CHKI-S or CHK1-L. Results are shown as the mean \pm standard error based on at least three independent experiments. (E\&F) Cell proliferations were assessed using EdU immunofluorescence staining in HepG2 (E) or QSG-7701 cells $(\mathrm{F}) . * P<0.05, * * P<0.01$ by Student's t-test.

To further investigate relationship of between CHK1-S or CHK1-L in tumor tissues and the prognosis of HCC patients, we made a survival analysis. Kaplan-Meier analysis revealed that both high CHK1-S and CHK1-L mRNA level in HCC tissues correlated with reduced RFS, but not in non-tumor hepatic tissues (Fig. 1D-G). Furthermore, we explored the relationship of the ratio of CHK1-S/L and the prognosis of HCC patients. As shown in Fig. 1H\&I, the group of patients with high ratio of CHK1-S/L in tumor tissues had a poor RFS than the group with low ratio, but not significantly in non-tumor hepatic tissues. Collectively, these data demonstrated that CHK1-S was associated with poor prognosis of HCC patients.

\subsection{CHK1-S significantly promoted the proliferation of HCC cells}

To investigate the biological functions of different CHK1 transcripts in HCC, we stably overexpressed CHK1-L or CHK1-S in HepG2 cells by transfecting CHK1-L expression vector (pLVX-CHK1-L) or CHK1-S expression vector (pLVX-CHK1-S) (as showed Fig. 2A). Cell Counting Kit-8 assays showed that ectopic expression of CHK1-S significantly accelerated HepG2 cell proliferation, and while overexpression of CHK1-L slightly promoted the cell proliferation (Fig. 2C). EdU 
incorporation assays also demonstrated that CHK1-S overexpressed HepG2 cells had more EdU-positive cells than CHK1-L overexpressed cells (Fig. 2D). Furthermore, colony formation assays showed the similar results (Fig. 2E). We also transiently transfected the indicated plasmids into QSG-7701 cells in order to overexpress CHK1-S and CHK1-L, respectively, and the EdU assay showed CHK1-S could accelerate QSG-7701 cell growth than the effect of CHK1-L, as same as the results of HepG2 cells (Fig. 2B\&2F). Collectively, these results demonstrated that CHK1-S significantly promoted HCC cell proliferation.

\subsection{SRPK I was associated with alternative splicing of CHK 1}

To investigate the mechanism underlying CHK1 splicing, we found some RNA binding protein genes (hnRNP A/B, RBM34, SRPK1, etc.) associated with gene alternative splicing were high expressed in HCC tumors through analyzing the microarray data (shown in supplementary table 1). Then we found that SRPK1, as an upstream kinase of splicing factor [20], was significantly higher in HCC tumors compared with matched non-tumor tissues both at the mRNA and protein levels (Fig. 3A\&3B). To explore whether the splicing process of CHK1-S is mediated by SRPK1, we transiently overexpressed SRPK1 in HepG2 and QSG-7701 cells, respectively. As shown in Fig. 3C, ectopic expression of SRPK1 significantly increased the protein level of CHK1-S. Besides, we found that SRPK1 mRNA expression levels were significantly correlated with CHK1-S mRNA levels in human HCC tissues (Fig. 3D). These data indicated that SRPK1 may be involved in the alternative splicing of CHK1.

\section{Discussion}

In the present study, we showed that CHK1-S was frequently overexpressed in HCC samples and high expression of CHK1-S and/or CHK1-L, and high ratio of CHK1 S/L in tumor tissue correlated with poor clinical outcome. Compared with CHK1-L, CHK1-S had stronger ability to promote cell proliferation. Furthermore, we found that SRPK1, as an upstream regulator of splicing factor, may be involved in regulating the splicing of CHK1-S.
A

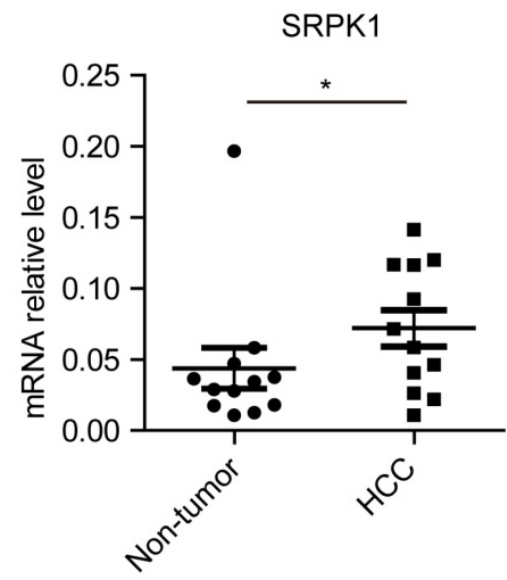

\section{B}

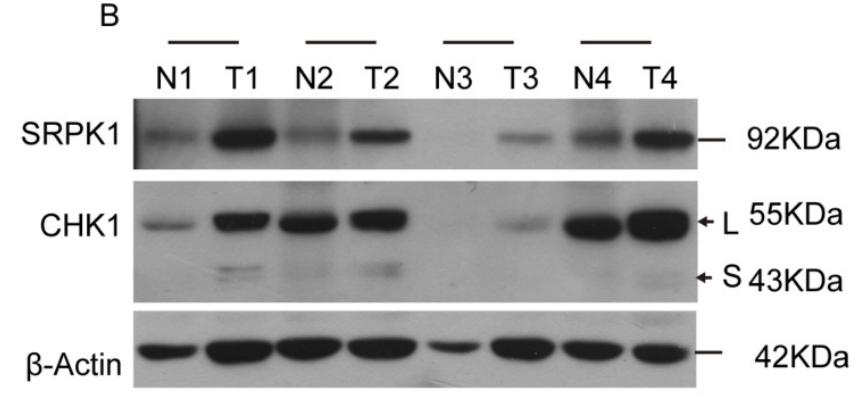

D
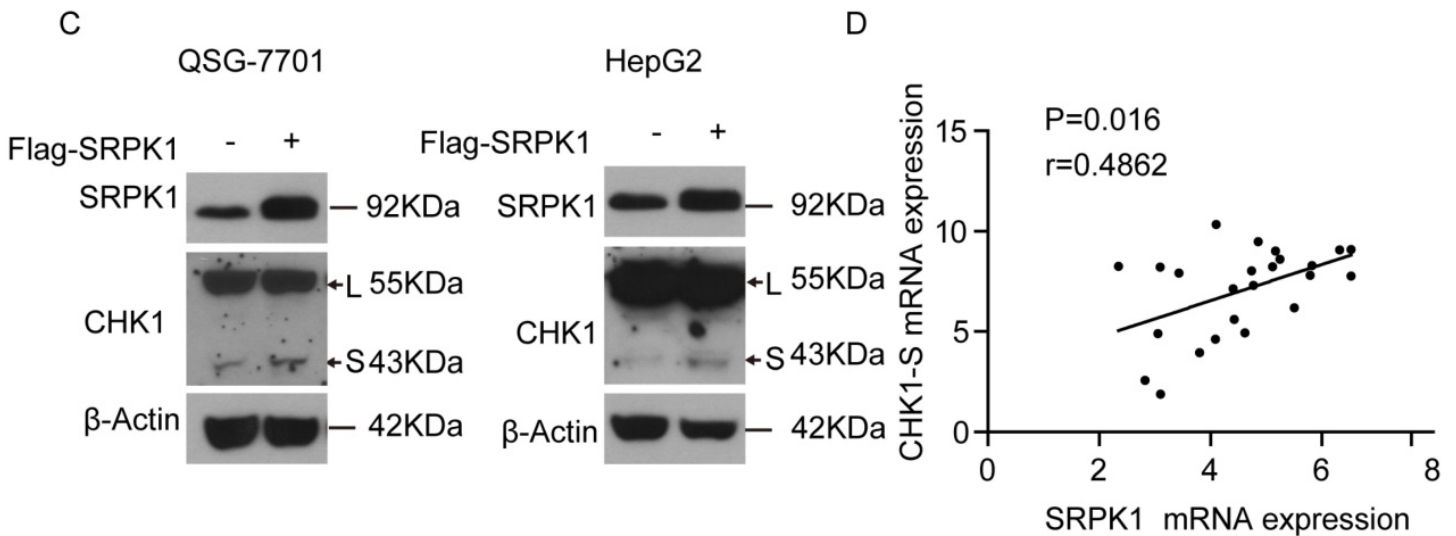

Figure 3. SRPK1 was associated with alternative splicing of CHK1-S. (A) SRPK1 mRNA levels in 12 paired HCC and adjacent non-cancerous hepatic tissues. $P$ values were acquired by Mann-Whitney test. Data are shown as median with interquartile range. (B) SRPK1 and CHK1 protein levels in 4 paired HCC and adjacent non-cancerous hepatic tissues. (C) Immunoblot analysis of CHK1-S (or CHK1-L) after transient overexpressing SRPK1 in HepG2 and QSG-7701 cells. (D) The correlation between CHK1-S and SRPK1 mRNA level in human HCC tissues $(n=24$ samples). $P<0.05, r=0.5807$ by Pearson correlation analysis. 
Table 3. Univariate and multivariate analysis of factors associated with overall survival of HCC patients.

\begin{tabular}{|c|c|c|c|c|}
\hline \multirow{3}{*}{ Variable } & \multicolumn{4}{|l|}{ Cox } \\
\hline & \multicolumn{2}{|l|}{ Univariate analysis } & \multicolumn{2}{|c|}{ Multivariate analysis } \\
\hline & HR(95\%CI) & $\mathrm{p}$ value & $\mathrm{HR}(95 \% \mathrm{CI})$ & $\mathrm{p}$ value \\
\hline Age & $0.991(0.968-1.016)$ & 0.483 & & \\
\hline Gender & $1.542(0.651-3.652)$ & 0.325 & & \\
\hline Tumor size & $1.109(0.981-1.254)$ & 0.097 & & \\
\hline $\begin{array}{l}\text { Microvascular } \\
\text { invasion }\end{array}$ & $3.954(2.010-7.779)$ & 0.000 & $3.929(1.959-7.879)$ & 0.000 \\
\hline Differentiation & $1.559(0.820-2.961)$ & 0.175 & & \\
\hline Satellite Nodules & $2.941(1.362-6.351)$ & 0.006 & & \\
\hline Envelope invasion & $1.924(0.920-4.022)$ & 0.082 & & \\
\hline Cirrhosis & $1.068(0.451-2.525)$ & 0.882 & & \\
\hline AFP & $1.052(0.959-1.153)$ & 0.284 & & \\
\hline $\begin{array}{l}\text { CHK1-S in tumor } \\
\text { tissue }\end{array}$ & $0.857(0.763-0.963)$ & 0.010 & & \\
\hline $\begin{array}{l}\text { CHK1-L in tumor } \\
\text { tissue }\end{array}$ & $0.894(0.765-1.044)$ & 0.157 & & \\
\hline $\begin{array}{l}\text { CHK1-S in adjacent } \\
\text { normal tissue }\end{array}$ & $0.918(0.842-1.001)$ & 0.053 & & \\
\hline $\begin{array}{l}\text { CHK1-L in adjacent } \\
\text { normal tissue }\end{array}$ & $0.923(0.816-1.044)$ & 0.200 & & \\
\hline $\begin{array}{l}\text { Ratio of CHK1-S/L in } \\
\text { tumor tissue }\end{array}$ & $0.694(0.540-0.890)$ & 0.004 & $0.685(0.524-0.897)$ & 0.006 \\
\hline $\begin{array}{l}\text { Ratio of CHK1-S/L in } \\
\text { adjacent normal } \\
\text { tissue }\end{array}$ & $0.786(0.630-0.982)$ & 0.034 & & \\
\hline
\end{tabular}

Many studies showed that the majority function of CHK1 was response to DNA damage, as a cell cycle checkpoint kinase. It induced cell cycle arrest in response to DNA damage mainly by phosphorylating Cdc25 family [21]. On the basis of these observations, CHK1 was initially thought to function as a tumor suppressor. However, numerous studies also suggest that $\mathrm{CHK} 1$ may actually promote tumor growth at least in some cancers [22-24]. Consistent with our results, CHK1 overexpression has been found in many tumors, such as T-cell acute lymphoblastic leukemia, triple-negative breast carcinoma [25, 26]. CHK1 may have oncogenic function in HCC, and is mainly detected in the cytoplasm of tumor cells [18]. Nuclear CHK1 prevents premature mitotic entry for monitoring of genomic integrity during cell proliferation [27]. CHK1 localizes in centrosome and interacts with UNC45A to regulate cancer cell proliferation [28]. So, the function of CHK1-S promotes cell proliferation perhaps not only as an activation inhibitor of CHK1-L but also having effect on the subcellular localization of CHK1-L. In our study, the high ratio of CHK1 S/L correlated with poor prognosis of HCC patients, consistent with the previous study that CHK1-S was up-regulated in testicular carcinoma tissues, especially in late-stage tumor samples [19]. High expression of CHK1-S could induce high genomic instability and high DNA-repair efficiency for survival in the unperturbed cancer cell, as previous study showed, CHK1 heterozygosity, conditional in the mouse mammary gland, resulted in three distinct haploinsufficient phenotypes that could contribute to tumorigenesis [29]. In addition, after comparing the expression of two CHK1 isoform, our data indicated that CHK1-S was just a spoiler, not the major factor for the progression of HCC (Fig. 1C, 3B).

SRPK1 can be activated by Akt and is an important branch to regulate alternative splicing [30]. Shuttling splicing factors are phosphorylated in the cytoplasm by SRPK1 and are subsequently transported to the nucleus [20, 31-33]. The serine/arginine rich (SR) proteins of splicing factors, such as SRSF1 which is a validated substrate of SRPK1 consequently increasing its nuclear activity and promoting target alternative splicing events, promote pre-mRNA splicing by binding to exonic splicing enhancers (ESEs) in pre-mRNA [34, 35]. Previous studies showed that Tra2 contained RS domain (domains enriched in arginine and serine residues) plays a role in regulating CHK1-S splicing [36, 37]. Our data showed that SRPK1 was associated with alternative splicing of CHK1 in both cancer cells and tumor tissues. Furthermore, SRPK1 had been reported to associate with hepatocellular carcinoma progression and poor patient survival [38]. Therefore, SRPK1 may mediate CHK1-S mRNA splicing through its downstream splicing factor in HCC. Our data also suggested that the SRPK1 protein may provide a novel target to inhibit HCC cell growth.

\section{Conclusion}

In summary, our study demonstrated that CHK1-S was highly expressed in HCC tissues and high CHK1-S expression was correlated with poor prognosis in HCC patients, indicating that CHK1-S could be a prognostic indicator of HCC and reducing CHK1-S expression may be a candidate target for HCC therapy. In addition, SRPK1 may be involved in this process of $\mathrm{CHK} 1$ splicing.

\section{Supplementary Material}

Supplementary figures and tables.

http://www.jcancer.org/v11p1792s1.pdf

\section{Acknowledgements}

This work was supported by the PUMC Fund of the Funds for the Central Universities (3332018193), National Natural Science Foundation (81641113), CAMS Innovation Fund for Medical Sciences (CIFMS) (2016-I2M-1-001).

\section{Competing Interests}

The authors have declared that no competing interest exists. 


\section{References}

[1] Bray F, Ferlay J, Soerjomataram I, et al. Global cancer statistics 2018: Globocan estimates of incidence and mortality worldwide for 36 cancers in 185 countries [J]. CA Cancer J Clin, 2018; 68: 394-424.

[2] Zheng R, Qu C, Zhang S, et al. Liver cancer incidence and mortality in china: Temporal trends and projections to 2030 [J]. Chin J Cancer Res, 2018; 30: 571-579.

[3] Yu SJ, Ma C, Heinrich B, et al. Targeting the crosstalk between cytokine-induced killer cells and myeloid-derived suppressor cells in hepatocellular carcinoma [J]. J Hepatol, 2019; 70: 449-457.

[4] Sveen A, Kilpinen S, Ruusulehto A, et al. Aberrant rna splicing in cancer; expression changes and driver mutations of splicing factor genes [J]. Oncogene, 2016; 35: 2413-2427.

[5] Climente-Gonzalez H, Porta-Pardo E, Godzik A, et al. The functional impact of alternative splicing in cancer [J]. Cell Rep, 2017; 20: 2215-2226.

[6] Kalsotra A, Cooper TA. Functional consequences of developmentally regulated alternative splicing [J]. Nat Rev Genet, 2011; 12: 715-729.

[7] Salton M, Misteli T. Small molecule modulators of pre-mrna splicing in cancer therapy [J]. Trends Mol Med, 2016; 22: 28-37.

[8] Chen J, Weiss WA. Alternative splicing in cancer: Implications for biology and therapy [J]. Oncogene, 2015; 34: 1-14.

[9] Castillo J, Goni S, Latasa MU, et al. Amphiregulin induces the alternative splicing of p73 into its oncogenic isoform deltaex 2 p73 in human hepatocellular tumors [J]. Gastroenterology, 2009; 137: 1805-1815 e1801-1804.

[10] $\mathrm{Lu} \mathrm{Y,} \mathrm{Xu} \mathrm{W,} \mathrm{Ji} \mathrm{J,} \mathrm{et} \mathrm{al.} \mathrm{Alternative} \mathrm{splicing} \mathrm{of} \mathrm{the} \mathrm{cell} \mathrm{fate} \mathrm{determinant}$ numb in hepatocellular carcinoma [J]. Hepatology, 2015; 62: 1122-1131.

[11] Luo ZL, Cheng SQ, Shi J, et al. A splicing variant of merlin promotes metastasis in hepatocellular carcinoma [J]. Nat Commun, 2015; 6: 8457.

[12] Zhang Y, Hunter T. Roles of chk1 in cell biology and cancer therapy [J]. Int J Cancer, 2014; 134: 1013-1023.

[13] Patil M, Pabla N, Dong Z. Checkpoint kinase 1 in DNA damage response and cell cycle regulation [J]. Cell Mol Life Sci, 2013; 70: 4009-4021.

[14] Dai Y, Grant S. New insights into checkpoint kinase 1 in the DNA damage response signaling network [J]. Clin Cancer Res, 2010; 16: 376-383.

[15] Blasius M, Forment JV, Thakkar N, et al. A phospho-proteomic screen identifies substrates of the checkpoint kinase chk1 [J]. Genome Biol, 2011; 12: R78.

[16] Harper JW, Elledge SJ. The DNA damage response: Ten years after [J]. Mol Cell, 2007; 28: 739-745.

[17] Chen Y, Poon RY. The multiple checkpoint functions of chk1 and chk2 in maintenance of genome stability [J]. Front Biosci, 2008; 13: 5016-5029.

[18] Hong J, Hu K, Yuan Y, et al. Chk1 targets spleen tyrosine kinase (1) for proteolysis in hepatocellular carcinoma [J]. J Clin Invest, 2012; 122: 2165-2175.

[19] Pabla N, Bhatt K, Dong Z. Checkpoint kinase 1 (chk1)-short is a splice variant and endogenous inhibitor of chk1 that regulates cell cycle and DNA damage checkpoints [J]. Proc Natl Acad Sci U S A, 2012; 109: 197-202.

[20] Giannakouros T, Nikolakaki E, Mylonis I, et al. Serine-arginine protein kinases: A small protein kinase family with a large cellular presence [J]. FEBS J, 2011; 278: 570-586.

[21] Mcneely S, Beckmann R, Bence Lin AK. Chek again: Revisiting the development of chk1 inhibitors for cancer therapy [J]. Pharmacol Ther, 2014; 142: 1-10.

[22] Brooks K, Oakes V, Edwards B, et al. A potent chk1 inhibitor is selectively cytotoxic in melanomas with high levels of replicative stress [J]. Oncogene, 2013; 32: 788-796.

[23] Xu J, Li Y, Wang F, et al. Suppressed mir-424 expression via upregulation of target gene chk1 contributes to the progression of cervical cancer [J]. Oncogene, 2013; 32: 976-987.

[24] Cole KA, Huggins J, Laquaglia M, et al. Rnai screen of the protein kinome identifies checkpoint kinase 1 (chk1) as a therapeutic target in neuroblastoma [J]. Proc Natl Acad Sci U S A, 2011; 108: 3336-3341.

[25] Sarmento LM, Povoa V, Nascimento R, et al. Chk1 overexpression in $\mathrm{t}$-cell acute lymphoblastic leukemia is essential for proliferation and survival by preventing excessive replication stress [J]. Oncogene, 2015; 34: 2978-2990.

[26] Verlinden L, Vanden Bempt I, Eelen G, et al. The e2f-regulated gene chk1 is highly expressed in triple-negative estrogen receptor / progesterone receptor / her-2 breast carcinomas [J]. Cancer Res, 2007; 67: 6574-6581.

[27] Matsuyama M, Goto H, Kasahara K, et al. Nuclear chk1 prevents premature mitotic entry [J]. J Cell Sci, 2011; 124: 2113-2119.

[28] Jilani Y, Lu S, Lei H, et al. Unc45a localizes to centrosomes and regulates cancer cell proliferation through chk1 activation [J]. Cancer Lett, 2015; 357: 114-120.
[29] Lam MH, Liu Q, Elledge SJ, et al. Chk1 is haploinsufficient for multiple functions critical to tumor suppression [J]. Cancer Cell, 2004; 6: 45-59.

[30] Zhou Z, Qiu J, Liu W, et al. The akt-srpk-sr axis constitutes a major pathway in transducing egf signaling to regulate alternative splicing in the nucleus [J]. Mol Cell, 2018; 71: 872.

[31] Lai MC, Lin RI, Tarn WY. Transportin-sr2 mediates nuclear import of phosphorylated sr proteins [J]. Proc Natl Acad Sci U S A, 2001; 98: 10154-10159.

[32] Ngo JC, Chakrabarti S, Ding JH, et al. Interplay between srpk and $\mathrm{clk} / \mathrm{sty}$ kinases in phosphorylation of the splicing factor asf/sf2 is regulated by a docking motif in asf/sf2 [J]. Mol Cell, 2005; 20: 77-89.

[33] Hamelberg D, Shen T, Mccammon JA. A proposed signaling motif for nuclear import in mrna processing via the formation of arginine claw [J]. Proc Natl Acad Sci U S A, 2007; 104: 14947-14951.

[34] Aubol BE, Wu G, Keshwani MM, et al. Release of sr proteins from clk1 by srpk1: A symbiotic kinase system for phosphorylation control of pre-mrna splicing [J]. Mol Cell, 2016; 63: 218-228.

[35] Lin JC, Lin CY, Tarn WY, et al. Elevated srpk1 lessens apoptosis in breast cancer cells through rbm4-regulated splicing events [J]. RNA, 2014; 20: 1621-1631.

[36] Best A, James K, Dalgliesh C, et al. Human tra2 proteins jointly control a chek1 splicing switch among alternative and constitutive target exons [J]. Nat Commun, 2014; 5: 4760.

[37] Long JC, Caceres JF. The sr protein family of splicing factors: Master regulators of gene expression [J]. Biochem J, 2009; 417: 15-27.

[38] Zhang J, Jiang H, Xia W, et al. Serine-arginine protein kinase 1 is associated with hepatocellular carcinoma progression and poor patient survival [J]. Tumour Biol, 2016; 37: 283-290. 